\title{
Directional Modulation Aided Secure Spatial Modulation
}

\author{
Qian Cheng ${ }^{\mathrm{a}}$, Jiang Zhu ${ }^{\mathrm{b},{ }^{*},}$, Junshan Luo ${ }^{\mathrm{c}}$, Zuohong Xu ${ }^{\mathrm{d}}$, and $\mathrm{Li}$ Zhu ${ }^{\mathrm{e}}$ \\ College of Electronic Science, National University of Defense Technology \\ 109\# Deya Road, Kaifu District, Changsha, P. R. China. \\ a chengqian14a@nudt.edu.cn, ", bjiangzhu@nudt.edu.cn, cluojunshan10@nudt.edu.cn, \\ dzuohong.xu@outlook.com, e zhulipaper16@163.com
}

\begin{abstract}
Keywords: Spatial modulation; directional modulation; physical layer security communications; near ML detector.
\end{abstract}

\begin{abstract}
A directional modulation (DM) aided secure spatial modulation (SM) scheme is proposed, which amalgamates the security of DM and high efficiency of SM, capable of achieving physical layer security (PLS) communications for SM. The principle of the proposed DM aided secure SM scheme is analyzed. Besides, the optimal maximum likelihood (ML) detector and a low-complexity near-ML (NML) detector are given for the receiver in the desired direction, respectively. Simulation results demonstrate that 1) the proposed DM aided secure SM scheme exhibits excellent BER performance at the desired receiver while the BER worsens at undesired receivers and 2) the NML detector reduces detecting complexity greatly with only $1 \mathrm{~dB}$ SNR penalty compared with the optional ML detector.
\end{abstract}

\section{Introduction}

Spatial modulation (SM), capable of achieving high channel capacity by introducing antenna index or spatial dimension to transmit information [1], has increasingly attracted researchers' interests in recent years.

Since the work in [1] proposed the concept of SM which is implemented at the transmitter side, it has been extended into various types. Specifically, a generalized SM scheme was proposed in [2] to transmit spatial information by activating multiple transmit antennas at each time slot. Then the SM technique was extended to receiver side in [3] by proposing precoding spatial modulation (PSM) and it was further generalized in [4]. The work in [5] put forward quadrature spatial modulation (QSM), which extended the SM constellation to in-phase and quadrature dimensions. Besides, the transmit-receive joint SM in [6] employed the indices of both transmit and receive antennas to transmit spatial information, and it was extended to generalized transmit-receive joint SM in [7]. The above-mentioned SM schemes can improve channel capacity to different extent. These SM schemes, however, mainly focus on the transmission efficiency without considering transmission security.

As a transmitter-side technique, directional modulation (DM) is capable of projecting digitally modulated information signals into a pre-specified desired communication direction while simultaneously distorting the constellation formats of the same signals in all other undesired directions [8], thus guaranteeing physical layer security (PLS) transmission. There are several methods to synthesize DM signals. The work in [9] utilized phased array to synthesize DM signals on radio frequency (RF) frontend. Afterwards, a low-complexity vector synthesis method for DM was proposed in [10] [11], which was synthesized on baseband. Afterwards, frequency diverse array (FDA) was introduced into DM to realize angle-range dependent DM. Specifically, the work in [12] utilized fixed frequency offsets-based FDA to synthesize angle-range dependent DM signals, and the angle-range dependent DM using time-modulated FDA was proposed in [13] [14]. These DM schemes, however, mainly focus on the transmission security without considering transmission efficiency.

Overall, both of SM and DM can be achieved using antenna arrays at the transmitter side, where the first mainly emphasizes transmission efficiency while the second primarily focuses on 
transmission security. The similarity between these two transmitter-side techniques naturally inspires us to propose a DM aided secure SM transmission scheme, which can exhibit high transmission efficiency and security. To the best of our knowledge, no work regarding the amalgamation of DM and SM is available in the present literature and we are the first to make this effort by proposing DM aided high-efficiency secure SM. The rest of this paper is organized as follows: Section II introduces the principles of the proposed DM aided secure SM scheme. Section III analyzes the maximum likelihood (ML) detector and further proposes a low-complexity near-ML detector. Section IV makes some simulations for the proposed DM aided secure SM scheme. Section V makes a conclusion.

Notations: Throughout the paper, superscripts $(\cdot)^{\dagger}$ and $(\cdot)^{*}$ denote pseudo inverse and conjugate transpose operations of a matrix, respectively. $\lfloor\cdot\rfloor$ refers to the floor operation. $|\cdot|$ and $\|\cdot\|$ denote the modulus of a complex number and the Frobenius norm of a vector respectively. Besides, $\mathbb{E}(\cdot)$ stands for the expectation operation of a random variable, and $\mathcal{C N}\left(0, \sigma^{2}\right)$ represents the complex Gaussian distribution with zero mean and variance $\sigma^{2}$.

\section{Principles of the Proposed DM Aided Secure SM System}

In this section, we will illustrate the principles of the proposed DM aided secure SM system in detail.

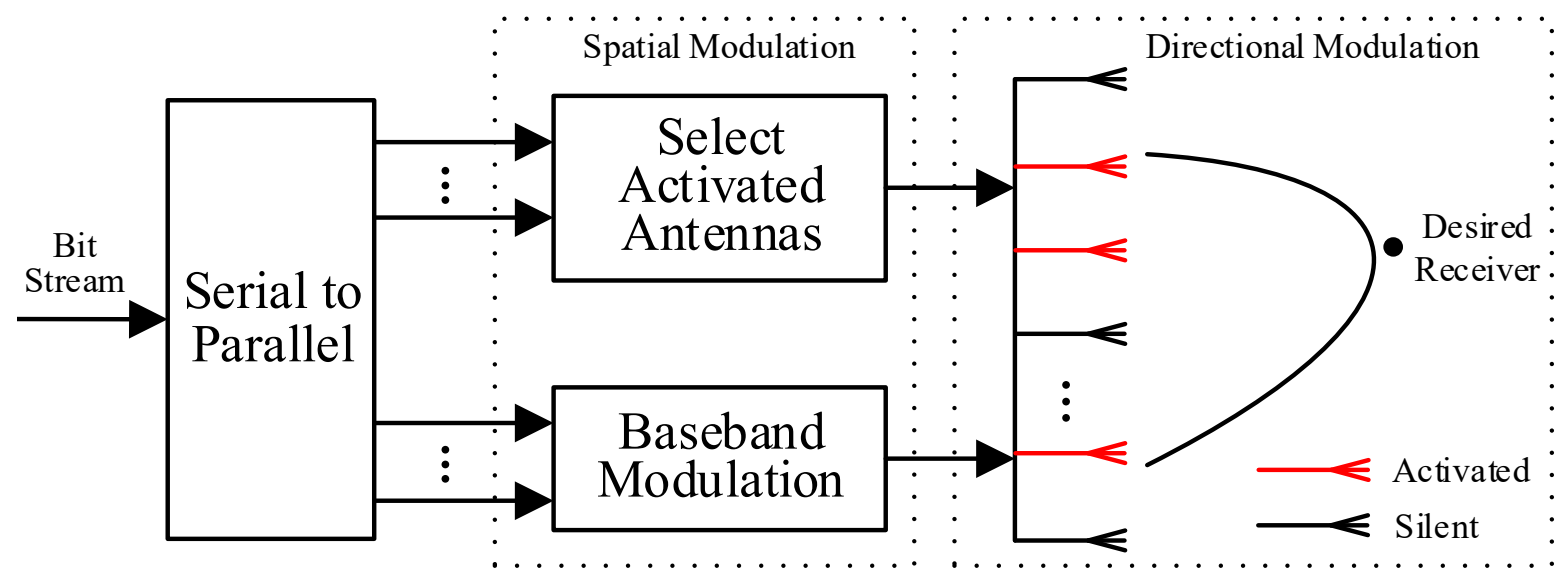

Figure 1. Architecture of the Proposed DM Aided Secure SM System.

As shown in Figure 1, we assume there are $N_{t}$ transmit and $N_{r}\left(N_{r}=N_{t}\right)$ receive antennas in the proposed DM aided secure SM scheme, where $N_{a}\left(1 \leq N_{a} \leq N_{t}\right)$ transmit antennas are activated at each time slot. Besides, the two-dimensional conventional signal constellation like phase shift keying (PSK) is employed, the alphabet of which is assumed to be $\mathcal{M}=\left\{s_{1}, s_{2}, \cdots, s_{M}\right\}$. Therefore, the number of bits transmitted at each time slot for the proposed DM aided secure SM system can be expressed as

$$
n=\left\lfloor\log _{2}\left(\begin{array}{l}
N_{t} \\
N_{a}
\end{array}\right)\right\rfloor+\log _{2} M
$$

Where $\left(\begin{array}{l}N_{t} \\ N_{a}\end{array}\right)$ and $\lfloor\cdot\rfloor$ denote combinatorial number and floor operations, respectively.

The bit block at each time slot is given as $B=\left[b_{0} b_{1} \cdots b_{n-1}\right]$, where the first $n_{1}=\left\lfloor\log _{2}\left(\begin{array}{c}N_{t} \\ N_{a}\end{array}\right)\right\rfloor$ bits, $b_{0} b_{1} \cdots b_{n_{1}-1}$, are used to select the combination index $i\left(1 \leq i \leq 2^{n_{1}}\right)$ of activated transmit antennas and 
further synthesize DM signals, and the last $n_{2}=\log _{2} M$ bits, $b_{n_{1}} b_{n_{1}+1} \cdots b_{n-1}$, are utilized to modulate the $m$-th baseband symbol $s_{m} \in \mathcal{M}, 1 \leq m \leq M$.

To synthesize a perfect standard constellation for the receiver in the desired direction, the low-complexity vector method for DM synthesis in [11] is introduced. The transmitting signal is designed as

$$
\mathbf{x}=\beta_{1} \sqrt{P_{s}} \mathbf{P}_{1} \mathbf{v}_{i} s_{m}+\beta_{2} \sqrt{P_{s}} \mathbf{P}_{2} \mathbf{z}
$$

where $P_{s}$ is the total transmit power constraint; $\beta_{1}$ and $\beta_{2}$ are the factors to split power, which satisfy the constraint $\beta_{1}^{2}+\beta_{2}^{2}=1 ; \mathbf{z}$ is the intentionally inserted complex artificial noise (AN) vector with each entry having zero mean and variance $\sigma_{z}^{2}$, i.e., $\mathbf{z} \sim \mathcal{C N}\left(\mathbf{0}_{N_{t} \times 1}, \sigma_{z}^{2} \mathbf{I}_{N_{t}}\right)$; and $\mathbf{v}_{i}\left(1 \leq i \leq 2^{n_{1}}\right)$ represents the $i$-th activated antenna combination vector with size $N_{t} \times 1$, where the antenna is activated if the entry equals 1 and silent if the entry equals 0 .

Besides, $\mathbf{P}_{1} \in C^{N_{t} \times N_{r}}$ in Eq. (2) is a normalization matrix with respect to the desired receiver's channel matrix $\mathbf{H}_{d} \in C^{N_{r} \times N_{t}}$. Given that the transmitter can acquire perfect feedback of channel state information (CSI) between the transmitter and the receiver in the desired direction, thus the normalization matrix $\mathbf{P}_{1}$ can be designed as the pseudo inverse of $\mathbf{H}_{d}$, that is,

$$
\mathbf{P}_{1}=\mathbf{H}_{d}^{\dagger}=\mathbf{H}_{d}^{*}\left(\mathbf{H}_{d} \mathbf{H}_{d}^{*}\right)^{-1}
$$

Where the superscripts $(\cdot)^{\dagger}$ and $(\cdot)^{*}$ denote pseudo inverse and conjugate transpose operations of a matrix, respectively. Evidently, the designed $\mathbf{P}_{1}$ satisfies

$$
\mathbf{H}_{d} \mathbf{P}_{1}=\mathbf{I}_{N_{r}} .
$$

Moreover, $\mathbf{P}_{2} \in C^{N_{t} \times N_{t}}$ in Eq. (2) is an orthogonal matrix with respect to $\mathbf{H}_{d}$. Considering the feedback of CSI, we can design $\mathbf{P}_{2}$ as

$$
\mathbf{P}_{2}=\mathbf{I}_{N_{t}}-\mathbf{H}_{d}^{*}\left(\mathbf{H}_{d} \mathbf{H}_{d}^{*}\right)^{-1} \mathbf{H}_{d}
$$

Which apparently satisfies orthogonality, i.e.

$$
\mathbf{H}_{d} \mathbf{P}_{2}=\mathbf{0}_{N_{r} \times N_{t}} .
$$

Generally, the line-of-sight (LoS) channel is considered in DM system, however, it cannot help recover the spatial or antenna index information of SM. Therefore, we will consider Rayleigh fading channel in this paper and introduce the vector synthesis method of DM to the proposed DM aided secure SM system.

After the transmitting signal passes through Rayleigh fading channel, the received signal at the receiver in the desired direction can be calculated by

$$
\begin{aligned}
\mathbf{y}_{d} & =\mathbf{H}_{d} \mathbf{x}+\mathbf{w}_{d} \\
& =\beta_{1} \sqrt{P_{s}} \mathbf{H}_{d} \mathbf{P}_{1} \mathbf{v}_{i} s_{m}+\beta_{2} \sqrt{P_{s}} \mathbf{H}_{d} \mathbf{P}_{2} \mathbf{z}+\mathbf{w}_{d} \\
& =\beta_{1} \sqrt{P_{s}} \mathbf{v}_{i} s_{m}+\mathbf{w}_{d}
\end{aligned}
$$


Where Eqs. (4) and (6) have been applied during the simplification process of Eq. (7). $\mathbf{w}_{d} \in C^{N_{r} \times 1}$ stands for additive white Gaussian noise (AWGN) vector which satisfies $\mathbf{w}_{d} \sim \mathcal{C N}\left(\mathbf{0}_{N_{r} \times 1}, \sigma_{\mathbf{w}_{d}}^{2} \mathbf{I}_{N_{r}}\right)$. Without loss of generality, the assumption of $N_{r}=N_{t}$ has also been considered in Eq. (7).

According to the uniqueness of channel, the CSI of an arbitrary eavesdropping receiver in the undesired direction is much different from the desired receiver in the desired direction and it is assume to be $\mathbf{H}_{u} \in C^{N_{u} \times N_{t}}$. Therefore, the received signal for the eavesdropper in the undesired direction can be expressed as

$$
\begin{aligned}
\mathbf{y}_{u} & =\mathbf{H}_{u} \mathbf{x}+\mathbf{w}_{u} \\
& =\underbrace{\beta_{1} \sqrt{P_{s}} \mathbf{H}_{u} \mathbf{P}_{1} \mathbf{v}_{i} s_{m}}_{\text {Distorted Signal }}+\underbrace{\beta_{2} \sqrt{P_{s}} \mathbf{H}_{u} \mathbf{P}_{2} \mathbf{z}}_{\text {Artificial Noise }}+\underbrace{\mathbf{w}_{u}}_{\text {AWGN }}
\end{aligned}
$$

Where the first term in Eq. (8) is distorted signal; the second denotes AN which further deteriorates the received signal for the eavesdropper in the undesired direction; and the third is AWGN which satisfies $\mathbf{w}_{u} \sim \mathcal{C N}\left(\mathbf{0}_{N_{u} \times 1}, \sigma_{\mathbf{w}_{u}}^{2} \mathbf{I}_{N_{u}}\right)$.

\section{Receiver}

We hope you find the information in this template useful in the preparation of your submission.

The maximum likelihood (ML) detector and a low-complexity near-ML detector for the desired receiver will be analyzed in this section.

\subsection{Detector}

The desired receiver needs to recover the bit information from its detected antenna combination index $\hat{i}$ and baseband symbol $\hat{s}_{m}$. From Eq. (7), we can generalize the maximum likelihood (ML) detector as

$$
\left\langle\hat{i}, \hat{s}_{m}\right\rangle=\arg \min _{1 \leq i \leq 2^{n_{1}}, 1 \leq m \leq M}\left\|\mathbf{y}_{d}-\beta_{1} \sqrt{P_{s}} \mathbf{v}_{i} S_{m}\right\|^{2}
$$

Where $\|\cdot\|$ denotes Frobenius norm operation.

Apparently, at least $2^{n_{1}} \cdot M$ times of Frobenius norm operations are required for the ML detector, the high complexity of which makes it unrealistic to realize the DM aided secure SM system. In order to reduce the detecting complexity, we further propose a low-complexity near-ML (NML) detector for the proposed DM aided secure SM.

\subsection{NML Detector}

Since the received signal of Eq. (7) at the receiver in the desired direction is scrambled only by AWGN, the NML detector can be calculated by two steps.

Firstly, detect the combination index $i$ of the activated antennas using Eq. (10):

$$
\hat{i}=\arg \min _{1 \leq i \leq 2^{n_{1}}}\left\|\left|\mathbf{y}_{d}\right|-\alpha \mathbf{v}_{i}\right\|^{2}
$$

Where $\alpha=\beta_{1} \sqrt{P_{s}} \mathbb{E}\left[\left|s_{m}\right|\right]$ is a deliberately multiplied threshold and $\left|\mathbf{y}_{d}\right|$ denotes the vector with each entry being the complex modulus of the corresponding entry of $\mathbf{y}_{d}$.

Secondly, detect the baseband symbol $s_{m}$ using the detected combination index $\hat{i}$ of the activated antennas using the following equation: 


$$
\hat{s}_{m}=\arg \min _{1 \leq m \leq M}\left\|\mathbf{y}_{d}-\beta_{1} \mathbf{v}_{\hat{i}} s_{m}\right\|^{2}
$$

From Eqs. (10) and (11), we can see that only $2^{n_{1}}+M$ times of Frobenius norm operations are required for the NML detector, which reduces the implementing complexity in a large scale.

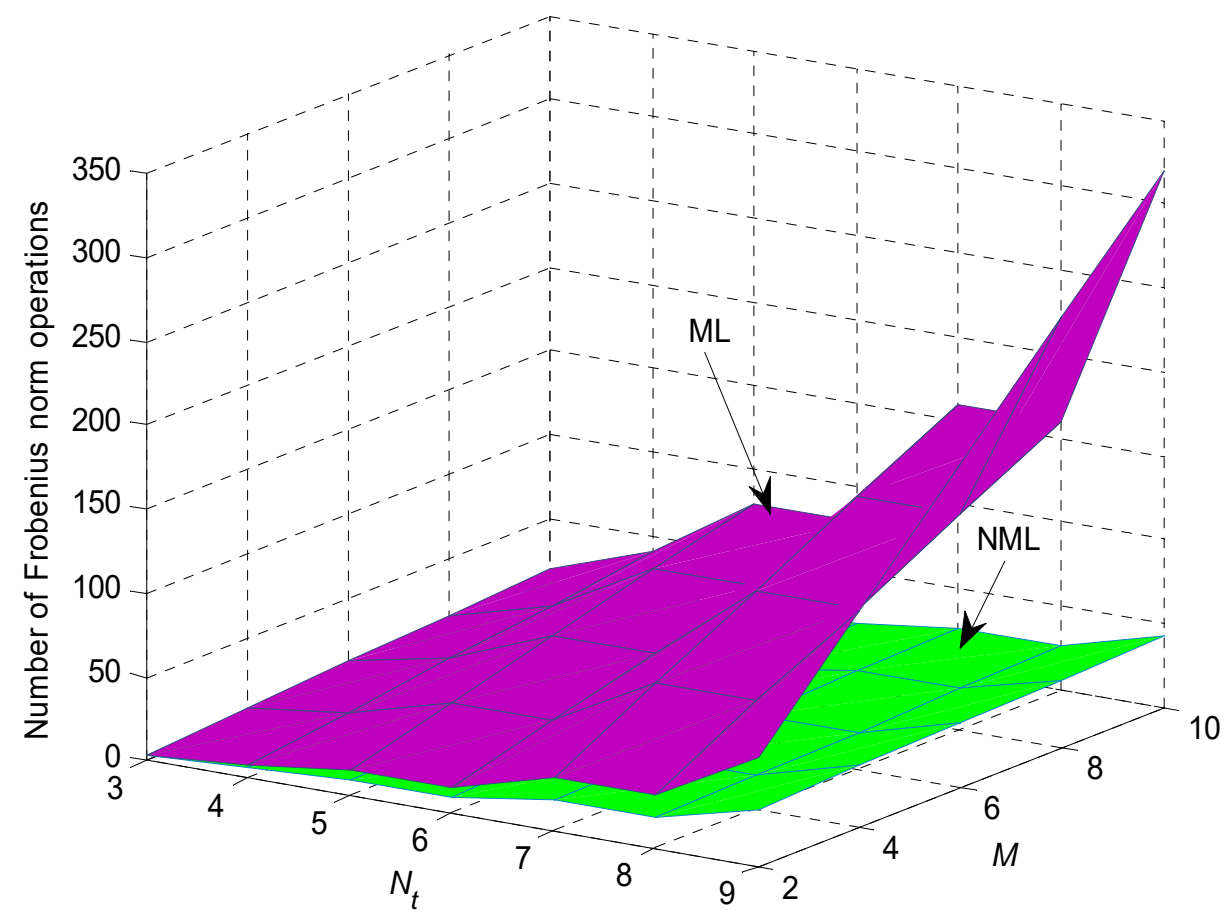

Figure 2. Complexity Comparison for the ML and NML Detectors. $\left(N_{a}=2\right)$

Figure 2 shows the complexity comparison for the ML and NML detectors in terms of the number of Frobenius norm operations. It is observed that the proposed NML detector can reduce the calculating complexity significantly, especially when the number of transmit antennas and the modulation order are large. The proposed NML detector, on the one hand, can greatly reduce the detecting complexity; on the other hand, it sacrifices the bit error rate (BER) performance in some degree, which will be discussed in the following section.

\section{Simulation Results}

In the simulations, we take $P_{s}=1, N_{t}=N_{r}=8$ and Gray-coded QPSK into considerations. Since the constellation of baseband symbols is normalized, i.e., $-\left[\left\|s_{m}\right\|^{2}\right]=1$, the signal-to-noise ratio (SNR) can be calculated by $\mathrm{SNR}=\beta_{1}^{2} P_{s} / \sigma_{\mathbf{w}_{d}}^{2}$, and we further assume $\sigma_{\mathbf{w}_{d}}^{2}=\sigma_{\mathbf{w}_{u}}^{2}$ without loss of generality. 


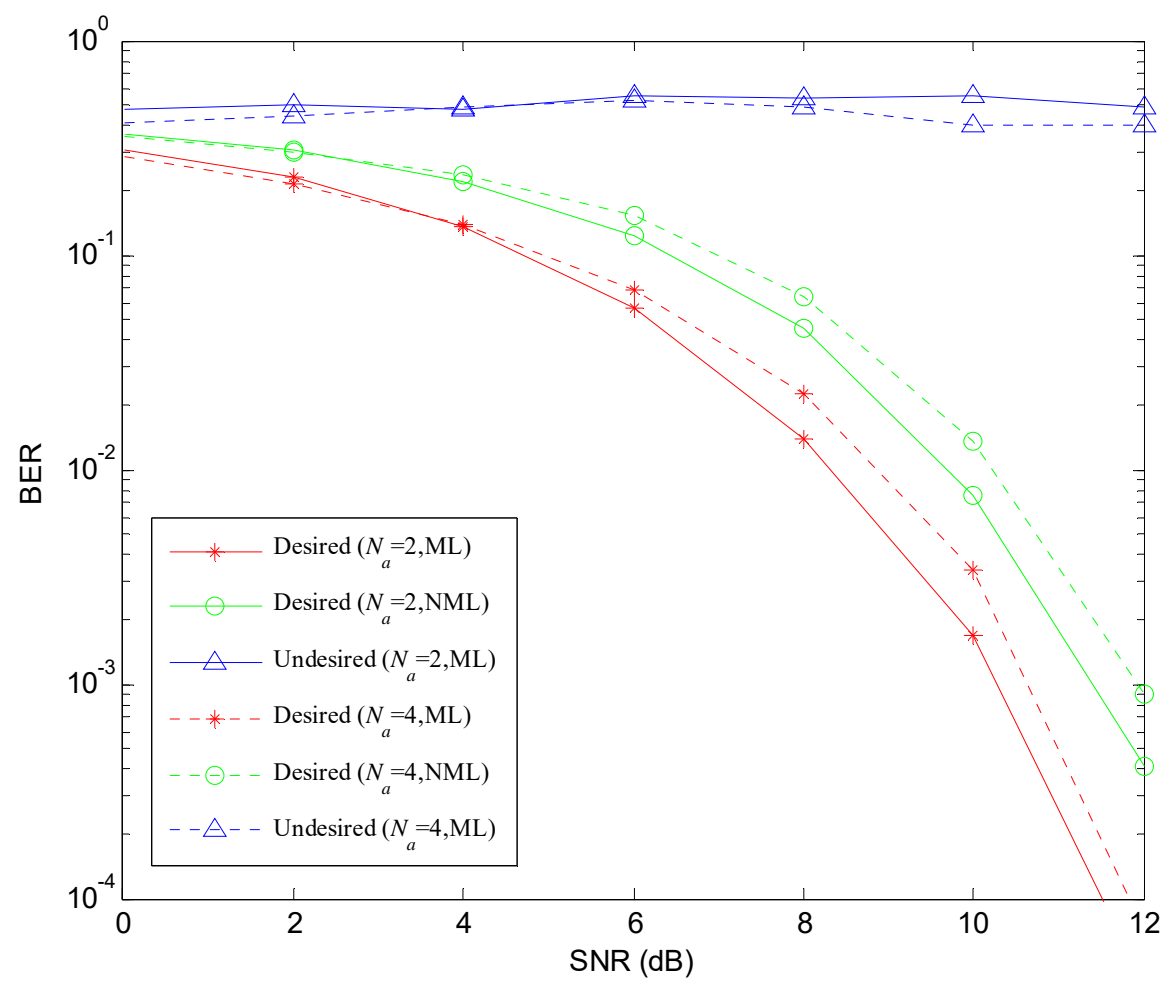

Figure 3. BER of the Proposed DM Aided Secure SM Versus SNR (dB). $\left(\beta_{1}^{2}=0.9\right)$

Figure 3 shows the BER performance versus SNR when $\beta_{1}^{2}=0.9$ and $N_{a}=2,4$. It can be seen that the BER of the receiver in the desired direction is much better than that of receivers in undesired directions. Besides, the BER performance is better when $N_{a}$ takes smaller number, which means that the number of the activated antennas, i.e., $N_{a}$, should be chosen reasonably by weighing good BER performance and high channel capacity. Moreover, seen from Figure 3, there is only about 1dB SNR penalty for the low-complexity NML detector to achieve the same BER as ML detector.

\section{Conclusion}

A DM aided secure SM scheme is proposed, which is capable of achieving secure and high-efficiency PLS communications. The principles of the proposed DM aided secure SM system are analyzed. The ML detector and a low-complexity near-ML detector are generalized, respectively. Simulations demonstrate that the proposed DM aided secure SM system behaves excellent BER performance at the desired receiver while the BER deteriorates at undesired receivers and the near-ML detector can reduces the calculating complexity significantly with a small penalty of SNR.

\section{References}

[1]. Raed M., Harald H., Sinanovic S., and et al. (2008) Spatial modulation. IEEE Trans. Veh. Tech., $57,4,2228-2241$.

[2]. Abdelhamid Y., Nikola S., Raed M., and et al. (2011) Generalised spatial modulation. Signals, Systems \& Computers, 45, 2, 1498-1502.

[3]. Lie-Liang Y. (2011) Transmitter preprocessing aided spatial modulation for multiple-input multiple-output systems. IEEE 73rd Vehicular Technology Conf. (VTC Spring), 1-5.

[4]. Rong Z., Lie-Liang Y., and Lajos H. (2013) Generalised pre-coding aided spatial modulation. IEEE Trans. Wireless Communications, 12, 11, 5434-5443. 
[5]. Raed M., Salama S. I., and Hadi M. A. (2015) Quadrature spatial modulation. IEEE Trans. Veh. Tech., 64, 6, 2738-2742.

[6]. Leijun W. and Xiao M. (2017) Coded transceive spatial modulation with block Markov superposition transmission. Electro. Lett, 53, 10, 657-659.

[7]. Qian C. and Jiang Z. (2017) Generalised transmit-receive joint spatial modulation. Electro. Lett, $53,24,1613-1615$.

[8]. Yuan D. and Vincent F. F. (2016) A review of directional modulation technology. Inter. Jour. Microw. \& Wirel. Tech., 8, 7, 981-993.

[9]. Michael P. D. and Jennifer T. B. (2009) Directional modulation technique for phased arrays. IEEE Trans. Ante. \& Prop., 57, 9, 2633-2640.

[10]. Yuan D. and Vincent F. F. (2014) A vector approach for the analysis and synthesis of directional modulation transmitters. IEEE Trans. Ante. \& Prop., 62, 1, 361-370.

[11]. Jinsong H., Feng S., and Jun L. (2016) Robust synthesis method for secure directional modulation with imperfect direction angle. IEEE Commun. Lett, .20, 1084-1087.

[12]. Wen-Qin W. (2017) DM using FDA antenna for secure transmission. IET Microw, Ante. \& Prop., 11, 3, 336-345.

[13]. Qian C., Jiang Z., Tao X., and et al. (2017) Time-invariant angle-range dependent directional modulation based on time-modulated frequency diverse arrays. IEEE Access, 5, 26279-26290, 2017.

[14]. Shaddrack Y. N., Wen-Qin W., and Jie X. (2017) Time-modulated FDA for physical-layer security. IET Microw, Ante. \& Prop., .11, 9, 1274-1279. 